\title{
Canine distemper virus isolated from a monkey efficiently replicates on Vero cells expressing non-human primate SLAM receptors but not human SLAM receptor
}

Na Feng ${ }^{1,2+}$, Yuxiu Liu ${ }^{3 \dagger}$, Jianzhong Wang ${ }^{1 \dagger}$, Weiwei $\mathrm{Xu}^{2}$, Tiansong Li ${ }^{4}$, Tiecheng Wang ${ }^{2}$, Lei Wang ${ }^{6}$, Yicong Yu ${ }^{2}$, Hualei Wang ${ }^{2}$, Yongkun Zhao ${ }^{2}$, Songtao Yang ${ }^{2}$, Yuwei Gao ${ }^{2^{*}}$, Guixue Hu ${ }^{1 *}$ and Xianzhu Xia ${ }^{1,2,5^{*}}$

\begin{abstract}
Background: In 2008, an outbreak of canine distemper virus (CDV) infection in monkeys was reported in China. We isolated CDV strain (subsequently named Monkey-BJ01-DV) from lung tissue obtained from a rhesus monkey that died in this outbreak. We evaluated the ability of this virus on Vero cells expressing SLAM receptors from dog, monkey and human origin, and analyzed the $\mathrm{H}$ gene of Monkey-BJ01-DV with other strains.

Results: The Monkey-BJ01-DV isolate replicated to the highest titer on Vero cells expressing dog-origin SLAM $\left(10^{5.2 \pm 0.2} \mathrm{TCID}_{50} / \mathrm{ml}\right)$ and monkey-origin SLAM $\left(10^{5.4 \pm 0.1} \mathrm{TCID}_{50} / \mathrm{ml}\right)$, but achieved markedly lower titers on human-origin SLAM cells $\left(10^{3.3 \pm 0.3} \mathrm{TCID}_{50} / \mathrm{ml}\right)$. Phylogenetic analysis of the full-length $\mathrm{H}$ gene showed that Monkey-BJ01-DV was highly related to other CDV strains obtained during recent CDV epidemics among species of the Canidae family in China, and these Monkey strains CDV (Monkey-BJ01-DV, CYN07-dV, Monkey-KM-01) possessed a number of amino acid specific substitutions (E276V, Q392R, D435Y and I542F) compared to the H protein of CDV epidemic in other animals at the same period.

Conclusions: Our results suggested that the monkey origin-CDV-H protein could possess specific substitutions to adapt to the new host. Monkey-BJ01-DV can efficiently use monkey- and dog-origin SLAM to infect and replicate in host cells, but further adaptation may be required for efficient replication in host cells expressing the human SLAM receptor.
\end{abstract}

Keywords: Canine distemper virus (CDV), Monkey, SLAM, H protein

\section{Background}

Canine distemper virus (CDV) is a single-stranded, negative-sense, nonsegmented RNA virus of genus Morbillivirus within the family Paramyxoviridae. CDV is a highly contagious pathogen that can cause disease with high morbidity and mortality in immunologically naive hosts as a result of viral tropism for the cutaneous,

\footnotetext{
* Correspondence:

gywtext@gmail.com; huguixue901103@163.com; xiaxzh@cae.cn

${ }^{\dagger}$ Equal contributors

${ }^{2}$ Military Veterinary Research Institute of Academy of Military Medical Sciences, Key Laboratory of Jilin Province for Zoonosis Prevention and Control, Changchun 130122, China

${ }^{1}$ College of Animal Science and Technology, Jilin Agricultural University, Changchun 130118, China

Full list of author information is available at the end of the article
}

respiratory, gastrointestinal, and central and peripheral nervous systems [1]. CDV has a broad host range and primarily affects animals belonging to the Canidae (e.g. dogs, wolves, and foxes) and Mustelidae (e.g. ferrets, badgers, and mink) families [2-4]. Previous studies had implicated CDV in the pathogenesis of Paget's disease [5], and natural CDV infection of non-human primates has been reported [6-8]. In 2006, a CDV outbreak occurred in rhesus monkeys (Macaca mulatta) at a breeding farm in Guangxi province, China, with a morbidity rate $(60 \%)$ and a mortality rate $(\approx 30 \%)$, unexpectedly [6]. Two additional $C D$ occurred in monkeys were reported in 2008. One occurred in rhesus monkeys at a laboratory animal center in Beijing with a reported $60 \%$ 
$(12 / 20)$ mortality [7], another occurred in Japan following the importation of cynomolgus monkeys (Macaca fascicularis) from China and was associated with a $10.6 \%(46 / 432)$ mortality [8].

Host cell infection initiates with viral binding to receptor proteins on the surface of cells. The CDV viral envelope contains two integral glycoproteins, the hemagglutinin $(\mathrm{H})$ protein and fusion (F) protein. The $\mathrm{H}$ protein mediates the binding of the virus to the cell membrane, and the F protein serves to fuse viral and host membranes, thereby enabling release of the viral contents into the cytoplasm [2]. The CDV-H glycoprotein mediates viral attachment through specific interactions with signaling lymphocyte activation molecule (SLAM) [9] or nectin-4 cellular receptors $[10,11]$. SLAM is expressed on a subset of immune cells, while nectin- 4 is expressed on epithelial cells of various organs. Although SLAM is a main receptor for morbilliviruses, each morbillivirus preferentially uses the SLAM of its host animals, as the specific residues within SLAM responsible for mediating interactions with the CDV-H protein can vary by species [12]. The specificity of the CDV-H protein for the SLAM receptor imposes species barriers and is partly responsible for restricting CDV host range $[12,13]$. Nectin-4 is highly conserved among different animals [10]. Unlike the SLAM receptor, the characteristics of binding between nectin- 4 and CDV were unknown. In this study, we evaluated the replication capacity of a CDV isolate obtained from a naturally infected monkey on Vero cells expressing dog, monkey, or human SLAM receptor proteins to better understand how CDV-H protein receptor specificity affects host range restriction.

\section{Results}

Surface expression of dog, monkey, and human SLAM on Vero cells

Vero cells were transfected with expression plasmids to express the dog, monkey, or human version of the SLAM protein. The expression plasmids included an HA epitope tag to allow confirmation of the surface expression of the SLAM protein. After cells stably transfected with G418 had been selected, SLAM expression was examined by flow cytometry using an HA tagspecific monoclonal antibody. The results showed that dog, monkey, and human SLAM was expressed equally on the surface of the resulting cell lines $(46.5 \%, 45.7 \%$ and $45 \%$, respectively) but not on the empty vectortransfected Vero cells (Fig. 1). These cells were named Vero/DogSLAM, Vero/MonkeySLAM and Vero/HumanSLAM, respectively. For virus isolation study, these cells were passaged fewer than five times.

\section{Isolation of CDV from the lung tissue of a deceased rhesus monkey}

We used the Vero/DogSLAM cells to isolate CDV from lung samples collected previously from a deceased rhesus monkey that had displayed signs of $\mathrm{CD}$. Inoculation of the Vero/DogSLAM cells with supernatants from the homogenized tissue resulted in an obvious cytopathic effect (CPE), whereas no CPE was observed after four blind passages of the supernatants on the Vero cells (Fig. 2, upper panels). Nucleoprotein expression in the cells was analyzed by indirect immunofluorescence assay (IFA). The nucleoprotein antigen was detected in Vero/DogSLAM cells inoculated with homogenized tissue supernatants but was not detected the inoculated Vero cells (Fig. 2, lower panels). The viruses isolated from the inoculated Vero/DogSLAM cells were named Monkey-BJ01-DV.

\section{Phylogenetic analysis of the $\mathrm{H}$ protein of Monkey- BJ01-DV}

We sequenced the full-length $\mathrm{H}$ protein of the MonkeyBJ01-DV (GenBank accession number KM923900) and performed a phylogenetic analysis of this strain and others deposited in GenBank (Table 1). The MonkeyBJ01-DV was highly similar to the other CYN07-dV monkey strains (AB687720; $99.8 \%$; 1823/1824 nucleotides) and Monkey-KM-01 (FJ405224; 99.0 \%; 1807/1824
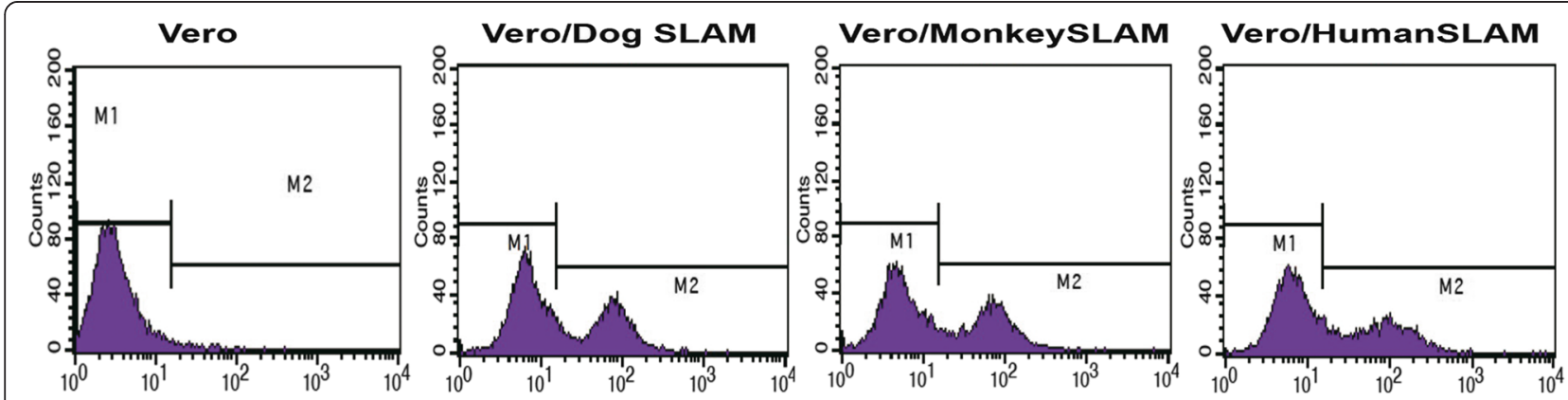

Fig. 1 Stable expression of dog, monkey and human SLAM protein on Vero cells. Vero cells were transfected with expression plasmids to mediate expression of HA epitope-tagged SLAM proteins from dog, monkey, and human and selected with G418. The control Vero cells were transfected by empty vector. The resulting cell lines were named Vero/DogSLAM,Vero/MonkeySLAM and Vero/HumanSLAM. Anti-influenza virus HA epitope MAb was used to confirm surface expression of each version of the SLAM protein by flow cytometry 


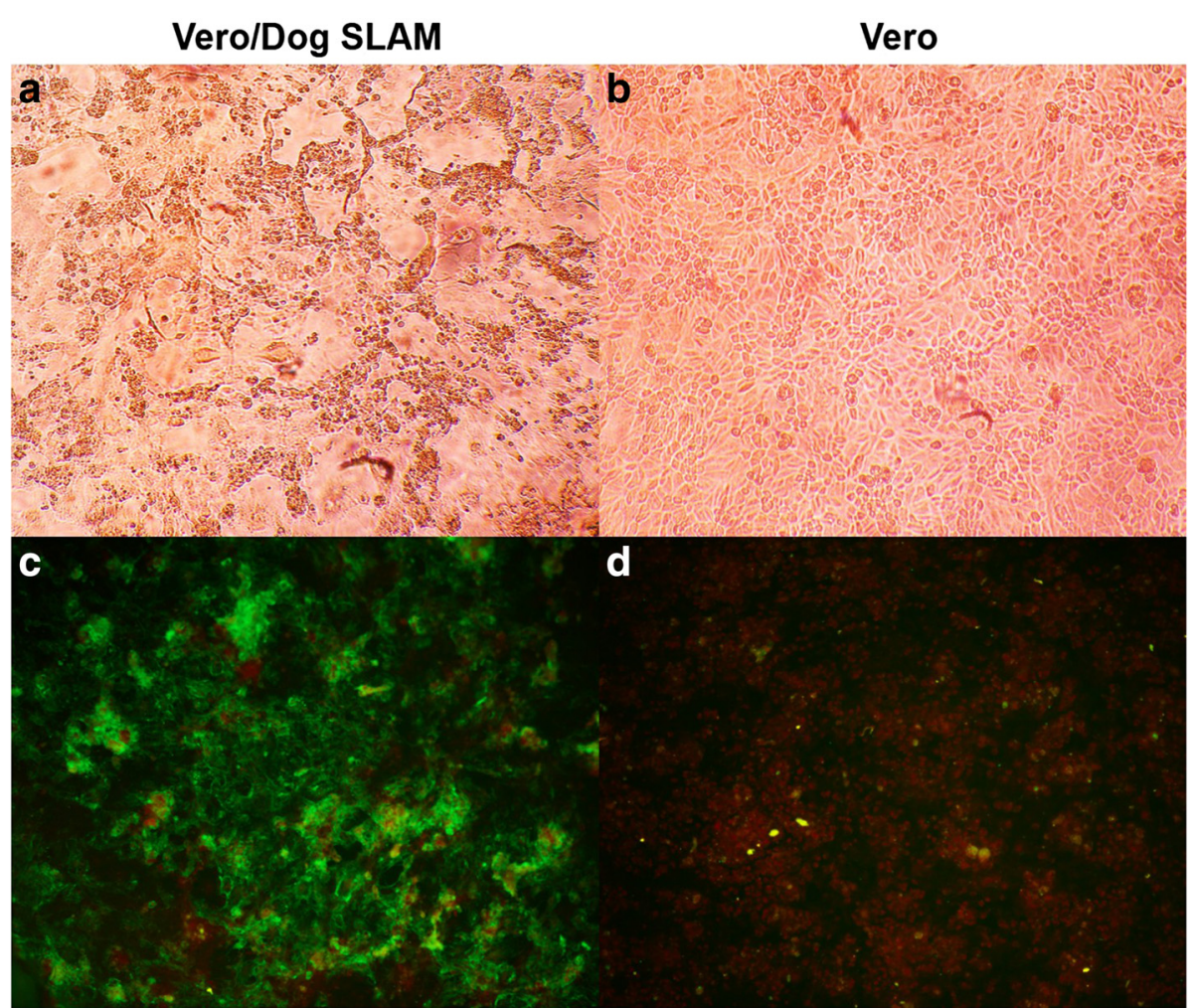

Fig. 2 Infection of Vero cells expressing dog SLAM protein with CDV from a deceased rhesus monkey. Microscopic evaluation of Vero/DogSLAM cells (a) and Vero cells (b) following inoculation with Monkey-BJ01-DV at a MOI of 0.01. Inoculation of Vero/DogSLAM cells resulted in obvious CPE. Detection of CDV antigens in Vero cells expressing the dog SLAM protein (c) and untransfected Vero cells (d) $64 \mathrm{~h}$ following inoculation with Monkey-BJ01-CDV at a MOI of 0.01. Cells were fixed and probed with a mouse monoclonal anti-CDV nucleoprotein antibody. A FITC-conjugated goat anti-mouse secondary antibody was used for detection

Table 1 Amino acid differences between the hemagglutinin of Monkey-BJ01-DV and other CDV isolates

\begin{tabular}{|c|c|c|c|c|c|c|c|c|c|c|}
\hline Amino acid sequence identity $^{a}$ & Sequence no. & 24 & 276 & 365 & 392 & 435 & 530 & 542 & 549 & 597 \\
\hline $98.4 \%$ & JN896331 (PS-dog) & S & E & V & Q & D & G & I & Y & $\mathrm{R}$ \\
\hline $97.5 \%$ & AB286946 (MD231-dog) & b & b & A & b & b & b & b & b & b \\
\hline $98.4 \%$ & JQ732173 (LDH (06)-fox) & b & b & b & b & b & b & b & b & b \\
\hline $98.4 \%$ & FJ848530 (BJ080514-dog) & b & b & b & b & b & b & b & b & b \\
\hline $98.2 \%$ & EU325720 (fox-Hebei07) & b & b & b & b & b & b & b & b & b \\
\hline $98.0 \%$ & EU325721 (fox-HLJ07) & b & b & $\mathrm{b}$ & b & b & b & b & b & b \\
\hline $97.4 \%$ & EU325724 (mink-LN) & b & b & $\mathrm{b}$ & b & b & b & b & b & b \\
\hline $97.9 \%$ & EU325728 (raccoon-JL07) & b & b & $\mathrm{b}$ & b & $b$ & b & b & b & $b$ \\
\hline $97.5 \%$ & GQ332530 (dog-Wuhan) & b & b & b & b & b & b & b & b & b \\
\hline $97.3 \%$ & CDV-TM-CC & b & b & b & b & b & b & b & b & b \\
\hline -—- & Monkey-BJ01-DV & $\mathrm{F}$ & V & A & $\mathrm{R}$ & Y & b & $\mathrm{F}$ & b & $\mathrm{H}$ \\
\hline $99.8 \%$ & FJ405223 (monkey-BJ-01) & $\mathrm{F}$ & V & A & R & Y & b & $\mathrm{F}$ & b & b \\
\hline 99.8 \% & AB687720 (mCNY07-dV) & $\mathrm{F}$ & V & A & $\mathrm{R}$ & Y & b & $\mathrm{F}$ & b & $\mathrm{H}$ \\
\hline $99.0 \%$ & FJ405224 (monkey-KM-01) & b & V & $A$ & $\mathrm{R}$ & Y & b & $\mathrm{F}$ & b & b \\
\hline
\end{tabular}

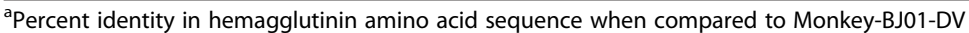

${ }^{\mathrm{b}}$ Indicate the same residues as the ones of JN896331 (PS-dog) 
nucleotides), which were isolated from a cynomolgus monkey and from a rhesus monkey, respectively $[6,8]$. These three monkey strains are each associated with CDV outbreaks among monkeys in China and belong to the Asia type I lineage. The CDV H protein is responsible for host cell attachment, is the most variable protein described for all members of the genus Morbillivirus and is an important determinant of the CDV host range [3]. We found that the $\mathrm{H}$ protein from these three strains of monkey-CDV possessed a number of amino acid specific substitutions compared to the $\mathrm{H}$ protein of CDV epidemic in other animals at the same period, as shown in Table 1: E276V, Q392R, D435Y and I542F. The glycine (G) residue at position 530 and the tyrosine $(\mathrm{Y})$ residue at position 549, which correspond to the partial SLAM-receptor binding region, were conserved among all three monkey CDV isolates and among CDV isolates from members of Canidae and Mustelidae (Table 1).

\section{Replication of Monkey-BJ01-DV on Vero cells expressing SLAM from dog-, monkey-, or human- origin}

We next evaluated the replication ability of the Monkey-BJ01-DV in engineered Vero/SLAM cell lines. Monkey-BJ01-DV replicated to the highest titers on Vero/DogSLAM $\left(10^{5.2 \pm 0.2} \mathrm{TCID}_{50} / \mathrm{ml}\right)$ and Vero/MonkeySLAM $\left(10^{5.4 \pm 0.1} \mathrm{TCID}_{50} / \mathrm{ml}\right)$ at $48 \mathrm{~h}$ post-infection, whereas replication on the Vero/HumanSLAM cells was reduced approximately 100 -fold $\left(10^{3.3 \pm 0.3} \quad \mathrm{TCID}_{50} / \mathrm{ml}\right.$; Fig. 3). The dog-CDV isolate from a Tibetan mastiff replicated to high titers on the Vero/DogSLAM cells and displayed reduced titers on the Vero cells expressing SLAM from monkey- or human- origin. Our results indicate that both the monkey- and dog-CDV isolates can efficiently replicate on Vero cells expressing either the monkey- or dog-origin SLAM receptor, but replicate less efficiently on cells expressing the SLAM receptor of human- origin.

\section{Discussion}

Morbilliviruses, including measles virus (MV), CDV, and rinderpest virus (RPV), are thought to have originated from a common ancestor several thousand years ago [14]. In general, however, each virus is able to naturally infect a relatively restricted number of host species. MV infection is limited to primates, CDV infection is limited to members of the Canidae, Mustelidae and Procyonidae families, and RPV infection is limited to ruminants [15]. Experimentally, monkeys are susceptible to MV infection and dogs to CDV infection [16]. Despite the apparent host range restriction of morbilliviruses, CDV has crossed species barriers, suggesting a potential ongoing expansion of host range. The first report of natural CDV infection of non-human primates was reported in 1989 among Japanese monkeys [15]. In 2006, a CDV epidemic affected a rhesus monkey (Macaca mulatta) breeding farm in China, inflicting high morbidity (60\%) and mortality $(\approx 30 \%)$ in young monkeys [6]. In 2008, Japan imported 432 cynomolgus monkeys (Macaca fascicularis) from China and 46 died from CDV while held in quarantine [8]. We isolated CDV from a deceased rhesus monkey (Monkey-BJ01-DV) following a 2008 outbreak in Beijing in which 12 of 20 rhesus monkeys died from CDV [7]. Collectively, these reports suggest significant changes in the epidemiology of CDV caused by an expanding CDV host range and/or viral virulence.

The complete genomes of three monkey-origin CDV isolates associated with outbreaks in China have been reported

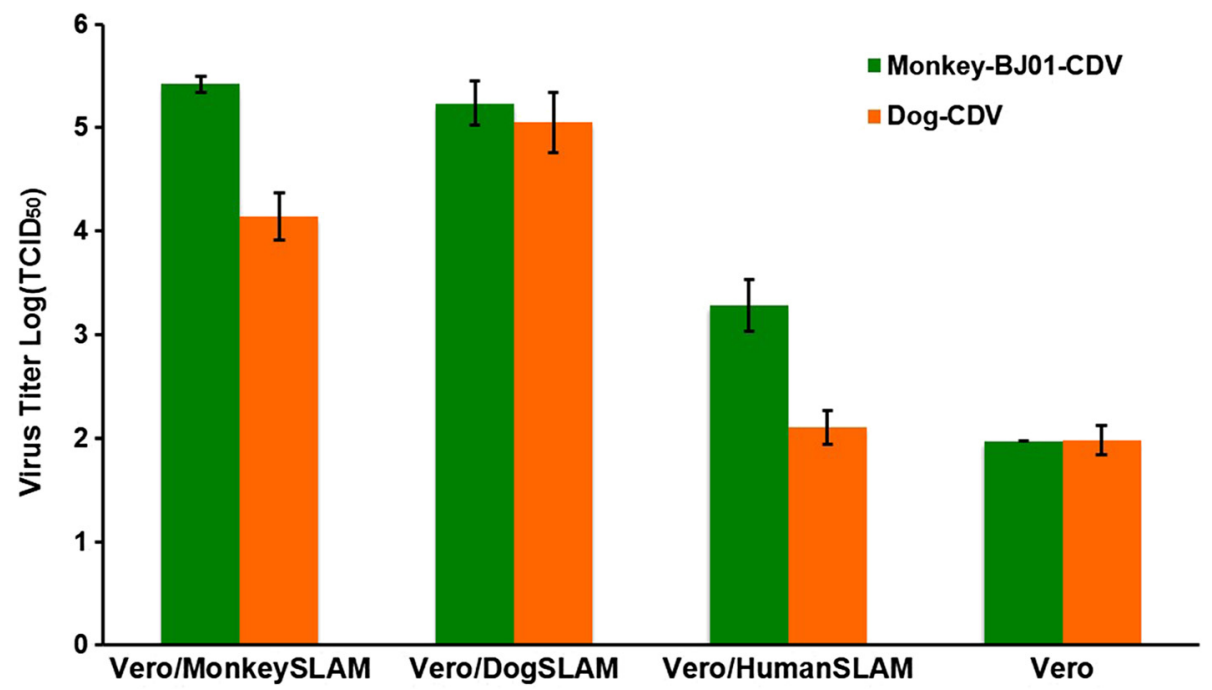

Fig. 3 Replication of Monkey-BJ01-DV and CDV-TM-CC in Vero/SLAM cells. Cells were infected at a MOI of 0.01. Viral titers were measured $48 \mathrm{~h}$ post-infection 
(Monkey-BJ01-DV (KF856711), CYN07-dV (AB687720) and MKY-KM-08 (HM852904) and are highly related to other epidemic CDV strains affecting species of the Canidae and Mustelidae families in China [6, 8] (Table 1). The $\mathrm{H}$ protein mediates viral attachment by binding to one or more cellular receptors $[9,11]$. Several studies have speculated about the impact of specific amino acid substitutions within the $\mathrm{H}$ protein on interactions with the SLAM receptor. In vitro receptor-binding studies showed that amino acid residues $527,528,529$, and 552 of the $\mathrm{H}$ protein are conserved among all morbilliviruses and are crucial for CDV-H to SLAM dependent fusion [4]. In particular, residues 530 and 549 both fall into receptor-binding domains located on propeller $\beta$-sheet 5 of the $\mathrm{H}$ protein [17]. Sequence analysis of the $\mathrm{H}$ gene of all three monkey CDV strains revealed a glycine $(\mathrm{G})$ and a tyrosine $(\mathrm{Y})$ at amino acid positions 530 and 549 of the partial SLAM-receptor binding region. G530 and Y549 are typically found in viral strains obtained from dogs in China, whereas other amino acid residues are present in CDV isolates obtained from wildlife $[18,19]$. This suggests that the CDV isolated from monkeys was transmitted from domestic dogs rather than from other wildlife. In this study, we found that amino acid Y549 was conserved within CDV lineages of the isolates analyzed, regardless of host species. CDV transmission in wild carnivore and non-canid species may most often occur between individuals within a species, and may also be influenced by a range of additional factors such as population size and ranging patterns. The three monkey CDV strains possessed E276V, Q392R, D435Y and I542F substitutions, which are unique changes when compared to the other Asia type I lineage strains. In particularly, the I542F substitution falls with the SLAM-binding regions of the $\mathrm{H}$ protein. The $\mathrm{H}$ protein amino acid substitutions identified among monkey CDV isolates may help explain recent changes in CDV host range.

The Monkey-BJ01-DV can efficiently grow on the Vero cells expressing SLAM from dog- and monkey-origin, but not the cells expressing SLAM receptor of human- origin. Interestingly, while the amino acid sequence identity of dog and monkey SLAM is only $63.6 \%$, the Monkey-BJ01DV strain can replicate on the Vero cells expressing SLAM receptor of dog-origin as efficiently as the Vero cells expressing monkey-origin SLAM. Factors other than the receptor binding, such as intracellular replication of the viruses may also be important for the establishment of infection. Further studies are required to understand the mechanisms by which CDV can cross species barriers.

\section{Conclusions}

Canine distemper virus isolated from a deceased rhesus monkey efficiently replicates on the Vero cells expressing non-human primate SLAM receptors but not human
SLAM receptor. The monkey origin-CDV-H protein could possess specific substitutions to adapt to the new host.

\section{Methods \\ Plasmids and cell lines}

Peripheral blood samples from a dog and rhesus monkey were collected from the Animal Hospital of Jilin University and the Animal Laboratory Center of the Academy of Military Medical Sciences, respectively. Human peripheral blood was kindly provided by the voluntary enrolled in this study. Peripheral blood mononuclear cells (PBMCs) of the dog, rhesus monkey and human were isolated from peripheral blood using dog and human lymphocyte separation media (TBD, Tianjin, China) according to the manufacturer protocols, respectively. Total RNA was extracted from the dog, rhesus monkey and human PBMCs after 2-4 h of stimulation with 2.5$3.0 \mathrm{mg}$ of phytohemagglutinin (PHA) per milliliter and was used for reverse transcription with oligo (dT) primers. The presence and location of signal peptide cleavage sites in the amino acid sequences from the dog-SLAM (AF325357), rhesus monkey-SLAM (XM001117605) and human-SLAM (NM-003037) were predicted by the Signal P 3.0 software. The SLAM gene of the dog, rhesus monkey and human without the signal peptide were encoded using various combinations of the primers designed on the basis of known SLAM sequences. The primer sets used were as follows: dogSLAM-F: 5' GCCTCGAGACAGGTGAGAGCTTGATGAAT-3' with the XhoI site underlined and dogSLAM-R: $5^{\prime}-\mathrm{G}$ CAGATCTTCAGCTCTCTGGGAACGTCAC-3' with the BglII site underlined for dog; monkey/humanSLAM-F: 5'-GCCTCGAGGCAAGCTATGGAACAGGTGGG-3'; monkeySLAM-R: 5'-GCAGATCTTCAGCTCTCTGGAAGTGTCACACT-3'; and humanSLAM-R: 5' TCAGATCTCTGGRARYGTCACRCT-3'), respectively. After checking against the NCBI reference sequence of dog SLAM, rhesus monkey SLAM and human SLAM, the cDNA encoding the DogSLAM, MonkeySLAM, and HumanSLAM proteins were inserted into the pCAGGS (Neo) vector possessing the immunoglobulin Igk leader sequence (GAG ACAGACACACTCCTGCTATGGGTA CTGCTGCTCTGGGTTCCAGGTTCCACTGGTGAC) and the influenza virus hemagglutinin (HA) epitope (TATCCATATGATGTTCCAGATTATGCT) [20], generated pCAGDogSLAM, pCAGMonkeySLAM and pCAGHumanSLAM. Vero cells constitutively expressing dog SLAM (Vero/DogSLAM), rhesus monkey SLAM (Vero/ MonkeySLAM) and human SLAM (Vero/HumanSLAM) were generated by transfecting Vero cells with pCAGDogSLAM, pCAGMonkeySLAM or pCAGHumanSLAM, respectively, and control Vero cells were transfected by empty vector. These cells were maintained in DMEM with $5 \% \mathrm{FBS}$ and $0.8 \mathrm{mg} / \mathrm{ml}$ Geneticin (G418; Invitrogen) in a 
humidified atmosphere at $37{ }^{\circ} \mathrm{C}$ and $5 \% \mathrm{CO}_{2}$. The above -described cells were stained with anti-influenza virus HA epitope MAb 12CA5 (Boehringer Mannheim), and then stained with fluorescein isothiocyanate (FITC)-conjugated goat anti-mouse IgG (Abcam, Cambridge science Park, UK). These stained cells were analyzed on a FACScan machine (Becton Dickinson) [2].

\section{Virus isolation}

Tissue samples used for virus isolation were obtained from the lungs of a deceased rhesus monkey during the CDV epizootic in Beijing in 2008 [7]. Lung tissue was suspended in cold phosphate- buffered saline (PBS) with antibiotics and was grinded into a homogenate. Homogenized lung samples were centrifuged at $2500 \mathrm{rpm}$ for $5 \mathrm{~min}$; the supernatant was collected and centrifuged an additional $5 \mathrm{~min}$ at $5000 \mathrm{rpm}$. Supernatants were inoculated onto monolayers of Vero/DogSLAM cells, from which a CDV isolate was subsequently obtained and named MonkeyBJ01-DV. An additional CDV-TM-CC strain was isolated from a Tibetan mastiff in our laboratory [21]. Normal Vero cells and Vero/DogSLAM cells were plated in 24well plates and infected with the Monkey-BJ01-DV. Productive CDV infection from the cultured cells was analyzed with a mouse monoclonal anti-CDV nucleoprotein antibody. A FITC-conjugated goat anti-mouse IgG was used as the secondary antibody, cells were analyzed with a fluorescence microscope (BX51FL; Olympus, Japan).

\section{RT-PCR and sequencing analysis of the Monkey-BJ01/ Monkey-BJ01-DV}

Total RNA was extracted from the lung of died monkey/ Monkey-BJ01-DV by using TRIzol reagent (Molecular Research Center Inc., USA) according to the manufacturer instructions. Reverse transcription (RT) was carried out using the Superscript II reverse transcriptase (Invitrogen, USA) according to the standard protocol. The CDV-H gene was cloned using the Ex-Taq DNA polymerase $(\mathrm{TaKaRa})$ with the following primers: CDVHF: GCGAATTCATGCTCTCCTACCAAGACAAGGTG with the EcoRI site underlined and according to the CDVHR: GGCCCTCGAGTCAAGGTTTTGAACGATTAC with XhoI site underlined. The PCR products were cloned into a pMD 18-T vector ( $\mathrm{TaKaRa}$ ) and were sequenced. At least five clones of each PCR products were analyzed to acquirie the accurate sequence. The sequence determined in this study had been registered at the GenBank under accession numbers FJ405223 and KM923900.

\section{Replication of CDV on Vero cells expressing the SLAM receptor from various animal species}

Vero/DogSLAM, Vero/MonkeySLAM, and Vero/HumanSLAM cells and Vero cells (empty vector transfected cells) $\left(1.8 \times 10^{5}\right.$ cells/well $)$ were cultured in 24 -well plates and infected with Monkey-BJ01-DV or CDV-TM-CC at a multiplicity of infection (MOI) of 0.01 . The cells and supernatants were harvested $48 \mathrm{~h}$ post-infection and were stored at $-80{ }^{\circ} \mathrm{C}$ until the virus titers were measured using the Vero/DogSLAM cells by the limiting dilution method and expressed as $\mathrm{TCID}_{50}$. Three independent experiments were performed to evaluate the CDV replication on the SLAM-expressing Vero cells.

\begin{abstract}
Abbreviations
CDV, canine distemper virus; CPE, cytopathic effect; F, fusion; FITC, fluorescein isothiocyanate; $\mathrm{G}$, glycine; $\mathrm{H}$, hemagglutinin; IFA, immunofluorescence staining; $\mathrm{MOI}$, multiplicity of infection; MV, measles virus; PBMCs, peripheral blood mononuclear cells; PBS, phosphate-buffered saline; PHA, phytohemagglutinin; $R P V$, rinderpest virus; $R T$, reverse transcription; SLAM, signaling lymphocyte activation molecule; $Y$, tyrosine
\end{abstract}

\section{Acknowledgements}

We thank Animal Hospital of Jilin University, Animal Laboratory Center of the Academy of Military Medical Sciences and the voluntary enrolled in this study for providing the peripheral bloods specimens. We also thank the Peter Wilker for editing the manuscript.

\section{Funding}

This work was supported by Chinese Special Fund for Agro-scientific Research in the Public Interest (201303042).

Availability of data and materials

Sequence data are deposited under GenBank accession numbers FJ405223 and KM923900.

\section{Authors' contributions}

$Y G$ and $X X$ designed and oversaw the experiments. NF, YL and JW wrote the manuscript. NF, TL, LW, XW and $Y Y$ carried out the laboratory experiments. TW, YL, HW and YZ analysed the data, interpreted the results. YG, SY and GH were involved in experimental design and manuscript revision. All authors have read and approved the submitted manuscript.

\section{Competing interests}

The authors declare that they have no competing interests.

Consent for publication

Not applicable.

\section{Ethics approval and consent to participate}

The protocol of the study was carried out in accordance with guidelines of animal welfare of World Organization for Animal Health. All experimental protocols were approved by the supervisory committee of science and technology of the Academy of Military Medical Sciences.

\section{Author details}

${ }^{1}$ College of Animal Science and Technology, Jilin Agricultural University, Changchun 130118, China. ${ }^{2}$ Military Veterinary Research Institute of Academy of Military Medical Sciences, Key Laboratory of Jilin Province for Zoonosis Prevention and Control, Changchun 130122, China. ${ }^{3}$ National Research Center for Veterinary Medicine, Luoyang, Henan 471000, China. ${ }^{4}$ College of Chemistry and Biology, Beihua University, Jilin 132013, China. ${ }^{5}$ Jiangsu Co-innovation Center for Prevention and Control of Important Animal Infectious Diseases and Zoonosis, Yangzhou 225009, China. ${ }^{6}$ Department of Animal Science and Veterinary Medicine, Henan Institute of Science and Technology, Xinxiang 453003, China.

Received: 12 August 2015 Accepted: 23 June 2016

Published online: 02 August 2016 


\section{References}

1. Osterhaus AD, de Swart RL, Vos HW, Ross PS, Kenter MJ, Barrett T. Morbillivirus infections of aquatic mammals: newly identified members of the genus. Vet Microbiol. 1995:44(2-4):219-27.

2. von Messling V, Zimmer G, Herrler G, Haas L, Cattaneo R. The hemagglutinin of canine distemper virus determines tropism and cytopathogenicity. J Virol. 2001;75(14):6418-27.

3. Pomeroy LW, Bjornstad ON, Holmes EC. The evolutionary and epidemiological dynamics of the paramyxoviridae. J Mol Evol. 2008;66(2):98-106.

4. von Messling V, Oezguen N, Zheng Q, Vongpunsawad S, Braun W, Cattaneo R. Nearby clusters of hemagglutinin residues sustain SLAM-dependent canine distemper virus entry in peripheral blood mononuclear cells. J Virol. 2005;79(9):5857-62.

5. Mee AP, Sharpe PT. Dogs, distemper and Paget's disease. BioEssays. 1993:15(12):783-9

6. Qiu W, Zheng Y, Zhang S, Fan Q, Liu H, Zhang F, Wang W, Liao G, Hu R. Canine distemper outbreak in rhesus monkeys, China. Emerg Infect Dis. 2011;17(8):1541-3.

7. Sun Z, Li A, Ye H, Shi Y, Hu Z, Zeng L. Natural infection with canine distemper virus in hand-feeding Rhesus monkeys in China. Vet Microbiol. 2010; 141(3-4):374-8.

8. Sakai K, Nagata N, Ami Y, Seki F, Suzaki Y, Iwata-Yoshikawa N, Suzuki T, Fukushi S, Mizutani T, Yoshikawa T et al. Lethal canine distemper virus outbreak in cynomolgus monkeys in Japan in 2008. J Virol. 2013;87(2):1105-14.

9. Seki F, Ono N, Yamaguchi $R$, Yanagi Y. Efficient isolation of wild strains of canine distemper virus in Vero cells expressing canine SLAM (CD150) and their adaptability to marmoset B95a cells. J Virol. 2003;77(18):9943-50.

10. Noyce RS, Delpeut S, Richardson CD. Dog nectin-4 is an epithelial cell receptor for canine distemper virus that facilitates virus entry and syncytia formation. Virology. 2013;436(1):210-20.

11. Pratakpiriya W, Seki F, Otsuki N, Sakai K, Fukuhara H, Katamoto H, Hirai T, Maenaka K, Techangamsuwan S, Lan NT et al. Nectin4 is an epithelial cell receptor for canine distemper virus and involved in neurovirulence. J Virol. 2012;86(18):10207-10.

12. Ohishi K, Ando A, Suzuki R, Takishita K, Kawato M, Katsumata E, Ohtsu D, Okutsu K, Tokutake K, Miyahara H et al. Host-virus specificity of morbilliviruses predicted by structural modeling of the marine mammal SLAM, a receptor. Comp Immunol Microbiol Infect Dis. 2010;33(3):227-41.

13. Nikolin VM, Osterrieder $\mathrm{K}$, von Messling V, Hofer H, Anderson D, Dubovi $\mathrm{E}$, Brunner E, East ML. Antagonistic pleiotropy and fitness trade-offs reveal specialist and generalist traits in strains of canine distemper virus. PLoS One. 2012;7(12), e50955

14. Norrby E, Sheshberadaran H, McCullough KC, Carpenter WC, Orvell C. Is rinderpest virus the archevirus of the Morbillivirus genus? Intervirology. 1985;23(4):228-32.

15. Yoshikawa Y, Ochikubo F, Matsubara Y, Tsuruoka H, Ishii M, Shirota K, Nomura Y, Sugiyama M, Yamanouchi K. Natural infection with canine distemper virus in a Japanese monkey (Macaca fuscata). Vet Microbiol. 1989;20(3):193-205.

16. DeLay PD, Stone SS, Karzon DT, Katz S, Enders J. Clinical and immune response of alien hosts to inoculation with measles, rinderpest, and canine distemper viruses. Am J Vet Res. 1965;26(115):1359-73.

17. McCarthy AJ, Shaw MA, Goodman SJ. Pathogen evolution and disease emergence in carnivores. Proc Biol Sci. 2007;274(1629):3165-74.

18. Muller A, Silva E, Santos N, Thompson G. Domestic dog origin of canine distemper virus in free-ranging wolves in Portugal as revealed by hemagglutinin gene characterization. J Wildl Dis. 2011;47(3):725-9.

19. Sekulin K, Hafner-Marx A, Kolodziejek J, Janik D, Schmidt P, Nowotny N. Emergence of canine distemper in Bavarian wildlife associated with a specific amino acid exchange in the haemagglutinin protein. Vet J (London, England : 1997). 2011:187(3):399-401.

20. Hara Y, Suzuki J, Noguchi K, Terada Y, Shimoda H, Mizuno T, Maeda K. Function of Feline Signaling Lymphocyte Activation Molecule as a Receptor of Canine Distemper Virus. J Vet Med Sci. 2013;75(8):1085-9.

21. Li W, Li T, Liu Y, Gao Y, Yang S, Feng N, Sun H, Wang S, Wang L, Bu Z et al. Genetic characterization of an isolate of canine distemper virus from a Tibetan Mastiff in China. Virus Genes. 2014;49(1):45-57.

\section{Submit your next manuscript to BioMed Central and we will help you at every step:}

- We accept pre-submission inquiries

- Our selector tool helps you to find the most relevant journal

- We provide round the clock customer support

- Convenient online submission

- Thorough peer review

- Inclusion in PubMed and all major indexing services

- Maximum visibility for your research

Submit your manuscript at www.biomedcentral.com/submit

) Biomed Central 